\title{
"To pay or not to pay» is not the only question
}

\section{Urs Brügger}

Prof. Dr. oec., Mitglied der Redaktion

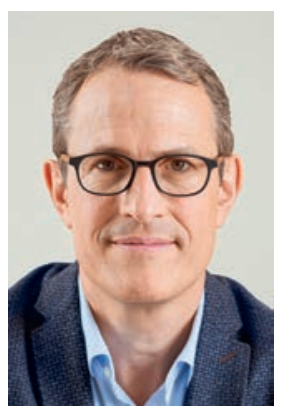

Das legendäre Myozyme-Urteil des Bundesgerichts aus dem Jahre 2010 enthielt bereits alle wichtigen Elemente des Dramas von heute um teure Medikamente für seltene und schwere Krankheiten. «Soll für Myozyme, dem damals einzigen zugelassenen Medikament für Morbus Pompe, eine schwere erblich bedingte und seltene Stoffwechselkrankheit, ein Preis von rund CHF 500000 pro Jahr von der Kasse bezahlt werden?», war die Frage. Der zu erwartende Nutzen war gemäss der damaligen dürftigen Studienlage bescheiden. Eine Patientin klagte; das Bundesgericht stützte letztinstanzlich den Entscheid der Kasse, die Kostengutsprache wegen mangelnder Wirtschaftlichkeit abzulehnen.

Die Problematik ist bis heute die gleiche. Sie wird im Beitrag von Jung et al. deutlich illustriert (siehe S. 1354 dieser Ausgabe). Die Autoren schlagen einen innovativen Lösungsansatz zum Art. $71 \mathrm{KVV}$ vor. Dieser soll Geschwindigkeit, Qualität und Fairness bei der Entscheidungsfindung verbessern, ohne das Kriterium der Wirtschaftlichkeit aus den Augen zu verlieren. Es lohnt sich, die drei zugrunde liegenden Themenfelder durch die gesundheitsökonomische Brille zu betrachten.

\section{1) Daten für fundierte Entscheide}

Wie schon der Myozyme-Fall gezeigt hat, ist die Datenlage für einen fundierten Entscheid in der Regel äusserst dürftig.

\section{2) Es fehlen Kriterien für die Unterscheidung wirtschaftlich/unwirtschaftlich}

Das Kriterium der Wirtschaftlichkeit im KVG bedeutet, dass eine medizinische Leistung nur vergütet wird, wenn sie als "wirtschaftlich» beurteilt wird. Das Bundesgericht begründete damals das Myozyme-Urteil mit gesundheitsökonomischen Studien, die eine Obergrenze von rund 100000 CHF pro gewonnenes Lebensjahr postulierten. Das Urteil erzeugte einen Aufschrei der Empörung. Paradoxerweise werden nun genau solche Richtgrössen (ICER-Werte) unter dem Titel «valuebased pricing" für die Gestaltung der Medikamentenpreise verwendet, was teilweise zu absurden Preisen führt. Die Diskussion über "wirtschaftlich» und «unwirtschaftlich» hat in der Schweiz noch nicht stattgefunden.

\section{3) "Not to pay» wird nicht verstanden und nicht akzeptiert}

Bei Medikamenten oder Indikationen, die nicht auf der SL geführt sind, müssen die Kassen gemäss Art. $71 \mathrm{KVV}$ die WZW-Kriterien im Einzelfall prüfen. Den Versicherern fällt dabei der Schwarze Peter zu bei der Beurteilung der Frage: «To pay or not to pay?» Doch es braucht die Möglichkeit, zum Entscheid zu kommen, dass ein Preis zu hoch ist. Nur so kann das solidarisch finanzierte Gesundheitssystem nachhaltig funktionieren. Wie Jung et al. mit ihrem Vorschlag aufzeigen, geht es eigentlich um die gleichen Probleme wie bei der Aufnahme neuer Arzneimittel in die SL. Die Autoren erwarten, dank Einbezug von Arzt und Patient die Datenlage bei umstrittenen medizinischen Leistungen zu verbessern. Sie fordern ein Pay-for-Performance-System. Dann könnte auch der Entscheid "not to pay» besser akzeptiert oder mindestens verstanden werden. Sie lehnen sich an das Verfahren des Coverage with Evidence Development (CED) an. CED bedeutet, dass eine Vergütung nur erfolgt, wenn vordefinierte Daten über die Indikationsqualität und den Nutzen der Therapie unter Einbezug von Daten aus Patient Reported Outcome Measures zwingend erfasst werden. Ein solches Register könnte den objektiven und subjektiven Patientennutzen systematisch erfassen, der Reduktion der Unsicherheit sowie als Grundlage für ein Pay-for-Performance-System und eine spätere Aufnahme in die SL dienen.

Ob der Vorschlag der Autoren praxistauglich ist oder nicht, wäre jetzt von Experten aus verschiedenen Perspektiven zu beurteilen. Sind ICER-Werte und valuebased pricing zielführend? Braucht es eine zentrale, objektive Beurteilungsstelle (Bund)? Kann es sein, dass ein einzelnes Medikament mehr kosten darf als der Aufwand des gesamten diagnostischen, behandelnden und betreuenden multiprofessionellen Teams zusammen? Sind behandelnde Ärztinnen und Ärzte, Patientinnen und Patienten objektiv genug? Die Diskussion ist angestossen, sie geht weit über die zystische Fibrose hinaus. 